\title{
MODÈLES TÉLÉVISUELS ET ÉVOLUTIONS DE LA PLACE DU POLITIQUE À LA TÉLÉVISION
}

\section{Frédéric Antoine ${ }^{1}$}

S'interroger sur la tendance à la spectacularisation de la présence du politique à la télévision est-il une préoccupation récente, liée aux révolutions auxquelles la télévision ne soumet ses téléspectateurs que depuis quelques années seulement ? Ou les rapports entre «politique » et «télévision » ne sont-ils pas de longue date problématiques et autant révélateurs de l'état d'esprit d'une société que des évolutions manifestes d'un média?

Il semble que, dans cette question comme dans d'autres, le point de vue de celui qui s'interroge influe sur la manière dont le problème se voit posé et sur la lecture qu'on en fait a priori. Préoccupé par un contexte déterminé dans le temps, on ne se soucie peut-être pas assez d'inscrire sa réflexion dans la durée, ni de la relativiser dans un modèle plus global. Or, ces deux démarches, permettant de percevoir la question de manière plus synoptique, procurent une opportunité de cadrer les choses quelque peu différemment.

On se préoccupera ici de saisir la question posée dans le contexte du paysage de la télévision en la Communauté française de Belgique, tout en concédant qu'il n'est pas possible d'aborder ce type de problématique dans un univers étriqué comme celui du Sud de la Belgique

1 Frédéric Antoine est professeur à l'École de Journalisme de Louvain (EJL) et membre de l'Observatoire du récit médiatique (ORM) au Département de communication de l'UCL.

Recherches en communication, $\mathrm{n}^{\circ} 24$ (2005). 
sans faire allusion à l'univers médiatique français, qui sert bien souvent chez nous d'exemple de référence.

\section{Le politique dans les médias audiovisuels}

Pour cadrer la question posée, il nous parait utile de remonter dans le temps. Car la télévision d'aujourd'hui n'est que le fruit des évolutions vécues depuis des décennies par ce média. Et les rapports qu'il entretient avec la chose politique sont quasiment aussi anciens que la télévision elle-même.

«Quasiment aussi anciens », écrivons-nous, car il importe de relativiser l'ancienneté de cette apparente fraternité entre politique et télévisuel. Et il paraît utile de nuancer, de même manière, cette impression de « fraternité ».

Il faut peut-être rappeler que, bien avant la naissance de la télévision, le politique entretenait déjà des rapports complexes avec l'univers $\mathrm{du}$ radiophonique. Et que ces rapports posèrent eux aussi problème. Parce que, dès qu'ils ont eu conscience de l'importance des médias audiovisuels comme moyens de communication de masse, les personnages politiques n'ont eu qu'un souci : chercher à s'en emparer pour les instrumentaliser. Les mettre à leur service afin qu'ils assument le rôle de courroie de transmission entre leur monde, celui du politique, de la chose publique et de l'État, et la population.

De leur côté, les médias électroniques ont tenté, dès qu'ils ont eu conscience de ce problème, d'affirmer leur indépendance vis-à-vis du politique. Et de là est née la première d'une longue série de tensions.

On ne peut en effet nier que, de tout temps, l'un des premiers soucis du politique a été de publiciser l'action qu'il exerçait, cette « publicité » de ses actes, considérée au sens premier du terme, étant à ses yeux le meilleur garant de sa légitimité. Or, longtemps, la difficulté majeure empêchant l'avènement d'une société réellement démocratique fut l'incapacité de la sphère politique d'établir ce contact légitimateur avec le plus grand nombre. L'ère des médias de masse réduisit ce handicap. L'avènement des médias audiovisuels l'allégea encore davantage.

L'histoire de l'INR, ancêtre de la RTBF, est un parfait exemple de cette soumission des médias audiovisuels publics au bon vouloir du politique. A contrario, celle de la BBC, dès la nomination de son premier directeur général Lord Reith, constitue la démonstration d'une lutte pour l'autonomie du média vis-à-vis du politique. 
Tous les manuels d'histoire de la communication ne manquent jamais de rappeler que c'est par le truchement de la communication radiophonique que le président Roosevelt réussit à faire passer l'idée du « New Deal » auprès de la population américaine, au cours de longues causeries vespérales au coin du feu. En France, à la même époque, les hommes politiques du Front Populaire s'étaient résolus à faire reposer sur la radio leur stratégie de communication avec les masses qui les avaient élus. La presse écrite était en effet entre les mains de groupes et de familles associés à la droite, et donc hostiles aux idées du Front. La radio, par contre, dépendant de l'État, avait tout pour servir d'outil de médiation au gouvernement au pouvoir...

Le contrôle strict que les États européens imposèrent aux médias audiovisuels au sortir de la guerre de 1940, les plaçant sous un régime de monopole généralisé, démontre également l'importance, mais aussi le type de considération, que la classe politique accordait alors aux mass-media de ce type.

Dans nos contrées, jusqu'aux années 1960, le politique estimait que les médias audiovisuels étaient en son pouvoir, et qu'il était de son droit d'en user comme bon lui semblait. Radio et télévision étaient considérées comme des «moyens » de communication, au sens propre du mot « moyen ». C'est-à-dire qu'il appartenait au politique d'y mettre un contenu, tandis qu'il relevait des «techniciens » des médias d'en assurer la transmission. Ce n'est qu'après le vote de la loi Harmel, en 1960, que radio et télévision ne dépendirent plus en Belgique du ministre des PTT, mais furent rattachés à celui de la culture... dont le poste venait seulement d'être créé.

En France, la littérature médiatique a démontré, si besoin en était, combien l'audiovisuel fut sous la coupe directe de la classe politique au pouvoir pendant une longue partie de la Ve République ${ }^{1}$. La tendance se renversera quand l'audiovisuel réussira à s'emparer d'une petite part d'autonomie vis-à-vis du politique et arrivera à marquer sa légitimité en cherchant à se libérer de son joug.

On célébrera en 2006 les cinquante ans du premier journal télévisé « national » diffusé en Belgique francophone. Mais il faudra davantage

1 On se rappellera par exemple de la phrase «Messieurs les censeurs, bonsoir», fameuse déclaration de Maurice Clavel quittant le plateau de l'émission À armes égales, sur l'ORTF en 1971 après qu'on avait coupé du commentaire d'un film qu'il devait présenter une phrase évoquant l'aversion du président Pompidou pour la résistance. 
de temps pour que la télévision publique gagne ses lettres de noblesse face au politique. Cette évolution est parfaitement décrite dans un ouvrage publié jadis à l'occasion des trente ans du journal télévisé de la $\mathrm{RTB}$, et qui retrace à merveille les premières années du rapport difficile et ambigu que le politique ne cesse d'entretenir avec la télévision.

L'histoire de la chronique politique au Journal Télévisé, c'est l'histoire d'un long parcours qui va du dédain à la passion. Au début des années 60 , dédain des hommes politiques pour le « cirque » télévisuel et dédain des journalistes pour le « théâtre » politique. À la fin des années 60, passion parfois excessive de l'homme politique pour une télévision qu'il voit comme unique source de succès ou d'échec, passion parfois aveuglante de certains journalistes pour les rebondissements d'une vie politique complexe, où les conflits se superposent et s'entrecroisent. ${ }^{1}$

Avant les années 1960, les professionnels des médias audiovisuels évitaient de traiter le politique. Déférence et distance étaient alors les maîtres mots du rapport entre les journalistes et la classe politique. « Il n'y avait que des déclarations. Pas d'interviews », explique un ancien journaliste de l'INR ${ }^{2}$. Le politique guidait la manœuvre et disait, quand il en avait envie, ce qu'il avait envie de livrer comme message.

L'appréhension de la gent journalistique vis-à-vis du monde politique aura la vie dure. Lorsque le Journal télévisé de la RTB prendra réellement forme, au début des années soixante, ses principaux acteurs se diront passionnés par l'actualité, mais certainement pas par la couverture de l'actualité politique nationale. Le domaine paraît tellement réservé que personne ne souhaite s'y frotter. On ne traitera donc cette actualité que par obligation, en ayant recours au laconisme de circonstance des dépêches de l'agence Belga et aux communiqués officiels, tout aussi froids et distants. Et aucune interview d'homme politique n'est sérieusement envisagée de pouvoir être réalisée « au débotté »3.

En Belgique francophone, les choses ne changeront que sous la pression d'un certain Frédéric François, qui se prendra au jeu du suivi de l'actualité politique et endossera vis-à-vis de la classe dirigeante l'attitude d'un «vrai » journaliste. Refusant d'aborder les personnages

1 LANOTTE M., DUPONT Ch., JESPERS J.-J., L'univers au jour le jour, Bruxelles, Crédit communal/RTBF éditions, 1986, p. 75.

2 Ibidem.

3 Ibidem. 
politiques en porte-micro, il se mettra à interpeller les hommes politiques à l'aide de questions directes, s'évertuant à les interroger comme le feraient les téléspectateurs. Sans langue de bois. Et en allant au centre de préoccupations concrètes.

C'est lors de l'apparition de ce type de journalistes que la classe politique s'est rendu compte qu'elle n'était plus maître du jeu de la communication médiatique, et que celui-ci était désormais orchestré par le média lui-même. Si le monde politique souhaitait toujours que le média se préoccupe de lui et répercute son image dans l'opinion, il y avait désormais lieu de se plier à ses conventions, à ses lois et à ses exigences.

Au tournant des années 1970, les hommes politiques se métamorphoseront devant la télévision, et apprendront à utiliser le média à leur avantage. Précurseur, le premier d'entre eux à suivre une formation à la communication télévisée sera Paul Vanden Boeynants. Il apprendra, notamment, à tenir compte de la caméra et à utiliser, mais pas toujours à bon escient, le fameux axe Y-Y qui permet à celui qui parle de s'adresser au téléspectateur « les yeux dans les yeux ». Sur cette lancée, les personnages politiques veilleront par la suite à leur présentation, mais aussi à l'adaptation de leur langage. À la télévision, ils comprendront petit à petit qu'ils ne s'adressent en effet plus à des médiateursjournalistes chargés de vulgariser leurs propos pour leurs lecteurs. Mais qu'ils parlent directement à l'opinion.

L'ouvrage sur le Journal télévisé déjà cité évoque cette transformation sur base d'une constatation identique faite en 1971 par un journaliste français : "À la télévision, il s'agit moins de convaincre que de persuader. L'argumentation elle-même a perdu de son prestige au profit de la "télégénie »: la télévision oblige l'homme politique à une simplification de son personnage et à une grande cohérence de ses attitudes. $»^{1}$

Peut-on considérer qu'il en est autrement, trente-cinq ans plus tard ? Les tendances amorcées au cours de la fin des années 1960 se sont affermies et se sont imposées avec d'autant plus de force que le média télévisé a, environ à la même époque, cliché sa grammaire et ses modes d'expression. Mais l'évolution était déjà en route, concrétisant cet « amour/haine » que les deux protagonistes entretiennent mutuellement l'un pour l'autre.

1 ROUARD J.-M., in Le Figaro, 28 septembre 1971, in LANOTTE M. et alii, Op. cit., p. 82. 


\section{Les émissions à contenu politique}

On vient de le dire : il fallut, avec le temps, s'adapter au média. La politique, pourtant, avait été présente à la télévision avant que la classe politique ne se soucie de mieux se conformer aux exigences du support. Dès les années soixante, les politiques avaient fait leurs premiers pas dans les studios. La télévision étant interdite d'entrée au Parlement, les hommes politiques avaient été invités sur les plateaux pour y reproduire, à l'échelon de la télévision, la substance du débat qui se déroulait dans les arènes politiques traditionnelles (Chambre ou Sénat).

Dans un premier temps, et la chose dura de longues années, le débat politique fut inséré à l'intérieur du cadre du journal télévisé. Mais on conçut aussi, assez rapidement, des émissions politiques indépendamment du rendez-vous quotidien d'informations. La véritable première émission politique de l'histoire de la RTB, Table ouverte, eut lieu le 25 janvier 1961. Elle inaugurait la première d'une longue série de débats dont la formule historique la plus marquante de l'histoire de la télévision belge fut Faire le point, qui démarra en juin 1965. Couplé avec Face à la presse, ces deux émissions politiques se fixèrent dans la case de programmation du dimanche midi et devinrent des rendez-vous tellement traditionnels que les programmes actuels qui en sont les héritiers, tant sur le service public que sur la chaîne privée, se déroulent toujours quarante ans plus tard dans la même grille de programmes du dimanche midi... Le succès relatif de ces programmes du dimanche midi fut tel, dès la fin des années 1960, que la présence de ce type d'émission à ce moment précis de la journée sera à l'origine des premiers bulletins d'informations de la RTB de la mi-journée, à partir de décembre 1969.

Quant aux soirées électorales, l'ouvrage sur l'histoire de l'information à la RTB en repère l'existence pour les élections du 26 mars 1961 déjà. La première « grande » opération liée à un scrutin aura lieu quatre ans plus tard. Et, dès 1968, la RTB elle-même considère que l'on peut parler lors d'élections d'une soirée " grand show électoral $»^{1}$, à l'image de ce que l'on recensait sur les autres télévisions occidentales.

1 Op. cit., p. 82. 


\section{La forme du politique}

«Grand show électoral ». Nous sommes en 1968 et le mot, déjà, est prononcé. Ne résonne-t-il pas toujours de belle manière dans la préoccupation de «spectacularisation» du traitement politique à la télévision qui nous occupe dans cette étude ? Or, il remonte à près de quarante ans...

Et son usage n'est pas innocent. Il atteste du fait que, immanquablement, tout traitement télévisuel d'une problématique amène, d'une manière ou d'une autre, à tenir compte des exigences de la mise en images, c'est-à-dire de la mise en spectacle. Et il est incontestable que cette tendance s'est affirmée au fur et à mesure que s'est imposé un langage télévisuel propre, conçu et réalisé par des professionnels de l'image et du son dont la première préoccupation n'était plus, comme à l'époque des pionniers, de parvenir à transmettre un signal électronique mais bien d'en construire le contenu en tenant compte de la spécificité du sport.

On a coutume de dire que la télévision est, au fil du temps, passée de l'état de "fenêtre ouverte sur le monde » à celui d'une "fenêtre ouverte... sur elle-même », ou à celui d'un « miroir »1. Au lieu d'aller à la rencontre du monde, elle aurait fini par se l'approprier et le reconstituer en ses studios. Afin de le (re)concevoir à l'échelle des exigences du média. Le politique n'a pas échappé à cette tendance générale. Naturellement, la télévision a entrepris de spectaculariser le politique parce que, au fil du temps, les hommes de télévision ont compris que ce média de l'image exigeait le recours à des procédés de visualisation qui imposaient une reconstruction particulière du réel.

Observer la configuration des plateaux de télévision au fil du temps constitue un bon indice de l'évolution de cette prise de conscience. Au dispositif «en rang d'oignon » des intervenants, au début des années 1960, s'est substitué un cadre d'échanges plus circulaire, proche du contexte d'une conversation de salon (ou de salle à manger). Et les transformations plus récentes ont tendu à modifier cette disposition pour la faire tendre vers celle de l'agora ou de l'hémicycle. Chacune de ces transformations a veillé à afficher le caractère interpersonnel, dialogué, des échanges, en abandonnant une mise en images «à plat».

1 D'où le titre du célèbre ouvrage de la sociologue française Dominique MEHL : La fenêtre et le miroir (Paris, Payot, 1992). 
Cette mise en à-plat, construite seulement à l'aide de face-caméras, tendait à confondre le statut du présentateur et celui de l'intervenant. Selon la grammaire de l'audiovisuel, l'axe Y-Y (« les yeux dans les yeux ») est d'abord l'apanage du présentateur, ou celui de l'énonciateur se trouvant en situation de communication directe avec le public des téléspectateurs. En tant que « personnages » participant à un programme dont ils sont les invités, les hommes (et les femmes) politiques n'ont normalement pas à recourir à ce mode d'adresse.

Mais il a fallu un certain temps avant que cette notion soit perçue par la classe politique ${ }^{1}$. Celle-ci n'avait, en effet, jamais saisi son rôle comme celui d'un simple intervenant participant à une mise en scène médiatique. À la tribune de la Chambre, dans un meeting électoral, les hommes politiques prenaient bien part à un certain cérémonial, mais ils s'adressaient directement à l'auditoire, en le regardant les yeux dans les yeux. La télévision exigeait, elle, le recours à une médiation supplémentaire. Du moins dans le cadre des programmes conçus par les professionnels du média.

Le monde politique eut donc (et a sans doute encore) la nostalgie de cette configuration d'adresse directe où, en cas de contexte extramédiatique, l'on sait à qui l'on parle parce qu'on le voit. Et où, dans le cadre d'une communication médiatique, on investit symboliquement l'objectif de la caméra comme étant la représentation symbolique de toutes les paires d'yeux de téléspectateurs présents derrière l'écran.

Longtemps, la télévision a d'ailleurs concédé aux «orateurs» (politiques ou autres) des lieux où ils pouvaient utiliser ce mode d'adresse : dans le cas des «tribunes libres » ou de ce que l'on appelle en Belgique les «émissions concédées ». Héritières de l'époque où le temps d'antenne de l'INR était en grande partie uniquement réparti entre les familles politico-philosophiques nationales, les «tribunes » répondent bien à leur définition : elles permettent à des individus de prendre la parole pour s'adresser à l'audience, de la même manière que l'orateur « montait » à la tribune de la Chambre ou accédait à celle du meeting de son parti pour parler à son auditoire. Dans ces tribunes médiatiques,

1 Cfr ci-dessus l'évocation de l'attitude de VDB qui, croyant jouer avec pertinence du média, avait pris la manie de s'orienter systématiquement vers la caméra dès qu'il répondait à une question, même s'il devait alors tourner le dos à son interlocuteur. La pratique plus récente de l'interview en amorce-épaule, qui s'est généralisée à la télévision, a permis de pallier cette difficulté en plaçant immédiatement les intervenants dans le bon axe. 
l'homme politique peut donc encore cultiver l'art qui semble lui être réservé : celui du discours ${ }^{1}$.

Le même dispositif prévaudra longtemps également pour les tribunes préélectorales. En cours de campagne électorale, l'opérateur audiovisuel est tenu de concéder du temps d'antenne à chaque parti. Et celui-ci l'occupe comme il l'entend, c'est-à-dire en faisant parler un par un, face caméra, ses principaux leaders.

Dans ce cadre énonciatif, l'homme politique semble se sentir plus à l'aise. Il opère seul, sur un terrain connu. Et la finalité du message reste inchangée : il s'agit de convaincre. Parler de convictions, d'idées. Argumenter pour défendre un point de vue. Des choses connues donc, qu'il suffit d'adapter formellement. Car, avec le temps, le politique comprendra que, devant une caméra, il doit modérer sa gestuelle, adoucir le ton de sa voix et éviter les effets de manche trop voyants.

Il faudra cependant se résoudre à comprendre que, même en adaptant légèrement la forme, l'expression politique éprouve des difficultés à passer la rampe télévisuelle. Pour s'en convaincre, il suffit de comparer les contenus récents des émissions concédées et des «tribunes électorales » avec ce qu'ils étaient jadis.

Que ce soit en radio ou en télévision, et même si la RTBF les présente toujours sous l'intitulé de «Tribunes concédées », les émissions concédées ne sont plus formellement conçues comme des tribunes précédées par la voix off d'un speaker annonçant de manière officielle et quelque peu pompeuse l'identité de l'intervenant. Dans la configuration la plus simple, un dialogue (au moins apparent ${ }^{2}$ ) a remplacé le monologue rhétorique jadis de mise. Il s'agit bien toujours de transmettre un message. Certains pensent toujours que celui-ci peut parvenir à convaincre. Mais l'habillage de la transmission s'est modifié pour se conformer au modèle dominant du média. Et, dans les cas les plus «médiatiquement construits », on ne recourt plus à de pseudo-inter-

1 Tous les hommes politiques ont commencé par investir les médias audiovisuels sous forme de discours, même dans le cas d'émissions qui ne permettaient pas la configuration de type « tribunes ». Dans leur ouvrage, LANOTTE et alii notent ainsi, à propos de la première diffusion d'un débat sur la RTB en 1961 : «La réalisation est statique, chacun est assis derrière une petite table portant une étiquette à son nom, Paul De Mol se borne à passer la parole. Mais en regardant ces émissions aujourd'hui, on ne peut s'empêcher de penser : quels orateurs!». (Op. cit., p. 78).

2 Il s'agit en effet très souvent de fausses interviews reposant sur des textes de questions/réponses rédigés par avance. 
views mais à la réalisation de véritables clips vidéo qui utilisent toute la palette des moyens et des modes d'écriture audiovisuels.

\section{La prise de parole à la télévision}

La manière d'assurer une prise de parole a donc évolué avec le temps, remisant à jamais hors des studios l'art du discours et les soliloques. La télévision dispose désormais elle-même des rênes de son destin. Elle règne sur son environnement et détermine elle-même les règles de son fonctionnement.

À la télévision, le dispositif énonciatif impose ainsi que toute parole soit nécessairement dialoguée. Et s'il reste çà et là une parole solitaire, elle est l'apanage des présentateurs, ceux-ci pouvant se permettre de déroger à la règle parce leur statut leur garantit de se trouver en permanence en situation de conversation symbolique avec l'auditoire. Or, ce statut n'est pas conféré aux « hôtes » de la télévision, c'est-à-dire à ceux qu'elle invite ou tolère dans ses émissions. Seules les situations de « confidence » permettent parfois à un intervenant télévisuel de s'exprimer seul en Y-Y face à une caméra ${ }^{1}$.

L'organisation du dialogue prend dès lors des formes multiples. Mais, à l'heure actuelle, elles reposent quasiment toutes, d'une manière ou l'autre, sur une mécanique d'interview. Selon le genre télévisuel utilisé, seule variera la place que prendra l'interviewer, et le rôle qui lui sera en définitive accordé dans le processus d'élaboration du produit télévisuel final.

Le dialogue prend évidemment place dans l'interview télévisée, qui constitue un genre journalistique majeur tant en ce qui concerne la récolte du matériel informationnel qu'en ce qui touche à son mode de communication. En comparaison avec l'autonomie d'expression que laisse la «tribune », on sait combien l'interview contingente la prise de parole, la cadre et l'organise. Elle en confère aussi la maitrise au questionneur, et non au questionné.

Mais elle permet notamment aussi à la télévision de maîtriser un élément essentiel de sa chaîne de production : la gestion du temps, question de plus en plus déterminante dans l'organisation du flot télévisuel. En accordant à l'interviewer la responsabilité de cadrer la prise de parole, la télévision s'arroge un meilleur contrôle sur l'écoulement

1 Situation vécue par tous les participants aux émissions de télé-réalité dans le « confessionnal » où ils sont invités à confier leurs états d'âme. 
de ses durées. Mais elle dépossède aussi l'interviewé d'une partie de sa liberté d'expression, lui imposant un contrat tacite selon lequel il accepte que le droit à un moment de parole sur le média vaille bien une concession concernant la durée de cette expression.

Cette domination de la parole recueillie par le truchement du questionnement a aussi sonné le glas d'une grande partie de ce qui relevait de la sphère du débat télévisé. Comme l'ont relevé de nombreux analystes, le débat repose normalement sur un échange d'opinions entre divers intervenants, échange dans lequel l'animateur ne remplit qu'un rôle de modérateur, sans prendre part au contenu de la discussion. Transposition du débat classique ayant lieu dans une arène politique ou dans un espace social défini, le débat télévisé a eu ses heures de gloire avant que la télévision ne s'approprie totalement les règles de sa grammaire et ne cherche à se rapprocher de son téléspectateur. Les premières émissions de la RTB que nous avons énumérées ci-dessus relevaient du débat politique.

Mais on perçoit que cette formule ne s'est rapidement pas avérée la seule possible, ni la meilleure télégéniquement parlant. Et qu'il était parfois plus avantageux de se rapprocher d'un « personnage » politique plutôt que d'en mettre plusieurs en opposition.

Le débat était un élément marquant de l'univers de la « paléo-télévision ». Au fur et à mesure que s'est imposée une « néo-télévision » ${ }^{1}$, de manière générale, le débat s'est vu remplacé par le talk-show, qui repose davantage sur une juxtaposition de prises de parole mises en scène par un animateur que sur l'organisation rationnelle d'un échange argumentaire. Tant et si bien qu'une part appréciable de la programmation des «moments de parole » à la télévision est aujourd'hui uniquement bâtie sur la logique du talk-show.

\section{La place du personnage politique}

Les différences marquantes entre le monde du débat et celui du talk-show se situent au niveau des contenus échangés, de la nature des échanges et du rôle que remplit face à cela l'animateur du programme. De manière simplifiée, on peut affirmer qu'un débat se définissait par la thématique qui y était abordée, alors qu'une émission de talk-show se repère d'abord en fonction de l'identité et de la personnalité de son

1 Même s'il ne faut pas accorder une importance trop déterminante au bornage temporel des époques distinguant ces deux genres. 
animateur. Cet élément illustre, sur un point précis, une des tendances essentielles de l'évolution du modèle télévisuel : un glissement des thèmes vers ceux qui les portent, à savoir les personnages. Et un glissement parallèle des contenus abstraits des thèmes vers leur matérialisation dans les expériences vécues par les personnages.

Le talk-show met en présence des individualités porteuses de cas concrets et de témoignages. Ceux qui y participent s'y rendent moins (sinon nullement) pour échanger des idées, des convictions ou des concepts, mais pour les matérialiser, au moins en partie par le truchement de leur vécu. Ce qui explique aussi pourquoi, dans un certain nombre de cas, les émissions de talk-shows mélangent les éléments de plateau avec des séquences de reportage, où les personnages présents en studio (c'est-à-dire en état d'extra-ordinarité) sont montrés en situation de quotidienneté.

Ces passages du rationnel énoncé au témoignage et de l'abstraction de l'idée à l'exemplification vécue constituent un des tournants majeurs de l'écriture télévisuelle, la télévision comprenant alors que, à l'instar de tous les arts de l'image, elle s'inscrit bien plus naturellement dans l'ordre du narratif que dans celui du discursif. Comme le démontrent les nombreuses analyses réalisées par l'Observatoire du récit médiatique de l'UCL depuis sa création, les médias audiovisuels sont même contraints de calquer d'abord leur mode de configuration sur le référent narratif. Et d'en exploiter les composantes naturelles, à savoir recourir à des personnages et à une mise en intrigue permettant d'ouvrir les possibles de l'histoire. On notera toutefois que cette intuition d'une «obligation de mise en récit» ne date pas d'hier. Si elle traverse incontestablement toute la télévision actuelle, ses prémices sont anciennes. En consultant les trois illustrations d'émissions politiques de la RTB que nous avons reproduites ci-dessus, on constatera par exemple que le titre de l'émission politique de 1977, Duel, ne manque pas de références à ce propos. Même s'il s'agit d'une confrontation politique, elle est conçue entre deux hommes qui incarnent deux politiques et deux univers antagonistes (Martens v/s Cools) et est bâtie sur le mode d'un affrontement d'homme à homme, à l'image d'un duel. L'intrigue en est évidente : elle pose dès le départ le contexte de la lutte et détermine la question qui tiendra le spectateur en haleine : qui remportera le duel, qui terrassera l'autre?

La personnification du traitement de la chose politique par la télévision peut dès lors être considérée comme un élément inhérent de l'être même du média télévisé. Elle appartient au schéma canonique 
de l'écriture télévisuelle. Et cet élément, lui non plus, n'est pas un fruit récent d'une évolution incontrôlée du média. Lorsque, en 1965, la RTB lance son programme Face à l'opinion, elle veille bien à inviter un homme politique afin qu'il réponde (ou dialogue) avec les questions des téléspectateurs. Il ne s'agit ni d'organiser une confrontation, ni de susciter un débat d'idées, mais d'entendre la parole d'un homme. Et si l'on consulte la liste des « grandes émissions politiques à la télévision » telle qu'elle a été dressée en France par l'INA, force est de constater que la majorité des programmes retenus ne relèvent pas, là aussi, du genre du débat, mais de celui de la rencontre ou de l'interview longue avec l'un ou l'autre personnage en vue. Au mieux relèvera-t-on que, dans certains cas, ce sont les interviewers qui sont en nombre, tandis que l'interlocuteur «mis sur la sellette» sera, lui, seul ${ }^{1}$. Et ce n'est qu'exceptionnellement que l'on rencontrera une confrontation « d'homme à homme », du type du Duel évoqué précédemment ${ }^{2}$.

Parmi les différentes configurations qui lui sont proposées, on peut considérer que ce mode individuel d'exposition de l'homme ou de la femme politique représente, mutatis mutandis, la situation télévisuelle actuelle dans laquelle il (ou elle) se sent la plus à l'aise. Ces situations permettent en effet au personnage politique de s'exposer comme il l'entend et de développer ses propos et ses idées. Il ne peut en faire autant dans des émissions de plateau de type talk-show ou dans des programmes où il n'est sollicité que de manière plus ponctuelle.

\section{Homme politique, vie publique, vie privée, médias}

Dès les années 1960, à la télévision, la vedettisation de la personnalité politique était déjà de mise. Elle ne fera naturellement que s'accroître. L'homme politique deviendra ainsi naturellement un «personnage » parmi d'autres au sein du monde médiatique.

1 En 1966 : Face à face se présente comme un débat entre un homme politique et des journalistes. En 1968, dans Face à la presse, une personnalité est interviewée par des journalistes. Dans L'heure de vérité (1972), une personnalité fait face à un journaliste et à d'autres intervenants. Sur TF1 en 1975, dans Questionnaire, une personnalité est interviewée sur des sujets d'actualités. Un seul invité aussi dans Cartes sur table (Antenne 2, à partir de 1977), dans Sept sur sept (TF1, à partir de 1981), puis dans la nouvelle formule de France 2 de L'heure de vérité, à partir de 1982.

21970 : À armes égales ou Les trois vérités (1973), l'un confrontant deux points de vue, l'autre mettant en débat un journaliste et deux personnalités. 
Le politique se voit ainsi incarné dans des individualités. Mais il est aussi devenu de plus en plus difficile aux individus concernés de gérer l'adéquation entre la représentation d'eux-mêmes générée par le média et le discours politique qu'ils entendent faire passer. Le point le plus profond du divorce entre télévision et classe politique se trouve sans doute de ce côté. Afin de rentrer dans les conditions d'accès au média télévisé, de nombreux hommes politiques se sont préoccupés de se bâtir un personnage et de conférer à cette identité médiatique suffisamment d'attraits différents pour remplir le plus souvent possible les conditions permettant de franchir le sas donnant accès à un traitement télévisuel. Mais une fois ce personnage bâti, les médias (et notamment la télévision) s'en sont emparés et l'ont gardé dans leurs filets.

Le personnel politique est dès lors devenu un personnage médiatique comme les autres, c'est-à-dire soumis aux règles du traitement réservé à tous les «personnages » réels que les médias révèlent à l'agenda de la société. La tendance a même été plus rapide et plus marquée en France qu'en Belgique. Une des premières manifestations de cette tendance sera, en 1985, l'apparition de l'émission Questions à domicile (TF1), où l'on cherchait à aller à la rencontre d'un homme politique dans son environnement et son cadre de vie quotidiens afin de mieux en percevoir la personnalité. Cette émission sera symbolique du rapprochement entre «vie privée » et «vie publique» du personnel politique.

On peut toutefois à nouveau s'interroger sur le caractère supposé spécifique à la sphère politique de cet intérêt du média télévisé pour côté « caché » de la vie des individus. Et, à nouveau, on risquera de conclure que ce mode d'approche de la chose politique se contente de s'inscrire dans le cadre de l'évolution générale du média, voire de l'ensemble de la société, où les frontières médiatiques séparant ce qui appartient au «privé » et relève du « public » ne cessent de se rétrécir. Ce que l'on nomme aujourd'hui «télé-réalité », mais qui ne constitue en fait qu'une étape dans un processus évolutif du modèle télévisuel, confirme à suffisance la perméabilité de plus en plus grande de ces frontières. À partir du moment où le personnage politique est considéré de la même manière que les autres personnages médiatiques, n'est-il pas normal qu'il soit soumis à un traitement similaire par la télévision ? Et ce d'autant plus aisément que, si le commun des mortels peut exprimer quelque réticence face à son exposition médiatique, il n'en est assurément pas de même de l'homme ou de la femme politique, qui est 
en général demandeur de présence télévisuelle pour les raisons déjà évoquées précédemment.

\section{Hommes politiques et peopleïsation médiatique}

Personnages médiatiques, les hommes et les femmes politiques bénéficiant d'une certaine notoriété sont, par la même occasion, devenus parties prenantes du processus de «peopleïsation » des personnages connus de la société dans laquelle ils évoluent. À nouveau, il ne s'agit pas là d'un processus purement télévisuel, mais plutôt du développement d'une mécanique médiatique générale reposant à la fois sur la place que les médias accordent aux acteurs de l'actualité, sur l'intérêt que le monde contemporain porte à la mise en récit de la vie de ses semblables et sur l'estompement progressif des barrières entre vie publique et existence privée.

Longtemps réservé aux personnages médiatiquement connus parce qu'appartenant aux mondes de la culture ou du show-business, le processus de «peopleïsation» des personnages médiatiques s'est étendu progressivement à d'autres sphères de la société. Le personnel politique a donc, naturellement, fait l'objet du même traitement, et ce avec d'autant plus de facilité que, dans la plupart des cas, il ne s'est pas opposé à ce processus et l'a même parfois encouragé.

On ne peut ainsi contester que les grands noms du personnel politique français appartiennent à l'univers des «people» d'OutreQuiévrain et que l'image de leur vie privée se mélange volontiers à celle de leur existence publique. Les rapports privilégiés que certains membres de la classe politique française entretiennent avec des acteurs de la scène médiatique télévisée, journalistique notamment, encouragent incontestablement ce phénomène.

En est-il de même en Belgique ? On ne peut parler dans notre pays d'une proximité aussi avouée entre le monde des médias et l'univers du politique. Mais il est incontestable que, depuis une dizaine d'années, le personnel politique en vue a, ici aussi, été englobé dans l'univers des « people» dont se soucient les médias.

Force est à nouveau de souligner qu'il ne s'agit pas à proprement parler d'un phénomène télévisuel, du moins dans le Sud du pays. On sait en effet que, dans le Nord, ce sont les chaînes de la télévision privées qui ont été à l'origine de la création des « B.V. », les « bekende Vlamingen ». Et que, assez naturellement, une partie de la classe poli- 
tique y a été associée même si, à l'origine, la grande majorité des B.V. flamands n'en étaient pas issus.

Nous aurions tendance à estimer que, dans le Sud, l'incorporation de la classe politique dans la catégorie des «people» n'est pas l'apanage de la télévision et qu'elle s'est médiatiquement réalisée «par défaut ». Ou, plus exactement, à défaut pour les médias de pouvoir recourir à d'autres personnages en vue. Historiquement, la partie francophone de la Belgique a toujours été en mal de vedettes populaires. Les stars francophones belges, comme la plupart de nos compatriotes qui ont réussi, n'ont acquis une certaine notoriété que par l'expatriation. Il faut au Belge francophone expérimenter la reconnaissance étrangère pour espérer bénéficier d'un minimum de sympathie dans son propre pays. De longue date, la presse populaire belge s'est heurtée à cette difficulté. Il y a une vingtaine d'années, l'édition francophone du magazine populaire Story s'est ainsi arrêtée faute de combattants. Et il suffit de faire un rapide survol des pages de couverture de La Libre Match pour tenter de circonscrire ce que l'on peut actuellement classer, en Belgique francophone, parmi les personnages médiatiques populaires méritant la «Une» du magazine qui se veut l'équivalent belge de Paris Match. Au terme de l'analyse, l'inventaire ne sera pas difficile à établir. Le panorama du monde people belge francophone comprendra la famille royale, quelques sportifs, très peu de vedettes de la chanson et du cinéma, un peu de personnages religieux et... des personnalités du monde politique ayant accepté de se départir d'une distance entre privé et public davantage de mise en Belgique qu'en France.

La télévision d'aujourd'hui n'est plus celle des débuts. Mais le média qui a commencé à s'installer dans le quotidien des Belges il y a cinquante ans possédait déjà, en germes, ce que l'on y trouve aujourd'hui. En ce qui concerne le traitement de la chose politique comme dans bien d'autres domaines. Afin de regarder clairement la situation actuelle, il nous semble que ce regard rétrospectif sur les évolutions du média télévisé, mais aussi sur l'ensemble des médias et la société belge, pouvait apporter une lecture plus relative et plus nuancée. 